\title{
Current oscillations generated by precipitate formation in the mixing zone between two solutions inside a nanopore
}

\author{
Erik C Yusko $^{1}$, Yazan N Billeh ${ }^{1}$ and Michael Mayer ${ }^{1,2}$ \\ ${ }^{1}$ Department of Biomedical Engineering, University of Michigan, 1101 Beal Avenue, \\ Lurie Biomedical Engineering Building, Room 2174, Ann Arbor, MI 48109-2110, USA \\ ${ }^{2}$ Department of Chemical Engineering, University of Michigan, 1101 Beal Avenue, \\ Lurie Biomedical Engineering Building, Room 2174, Ann Arbor, MI 48109-2110, USA \\ E-mail: mimayer@umich.edu
}

Received 12 May 2010, in final form 15 July 2010

Published 29 October 2010

Online at stacks.iop.org/JPhysCM/22/454127

\begin{abstract}
Unlike biological protein pores in lipid membranes, nanopores fabricated in synthetic materials can withstand a wide range of environmental conditions including the presence of organic solvents. This capability expands the potential of synthetic nanopores to monitor chemical reactions occurring at the interface between solutions of organic and aqueous character. In this work, nanopores fabricated in borosilicate glass or silicon nitride connected a predominantly organic solvent to an aqueous solvent, thereby generating a mixing zone between these solutions inside the pore. This configuration was exploited to precipitate small organic molecules with low aqueous solubility inside the nanopores, and concomitantly, to monitor this precipitation by the decrease of the ionic conductance through the nanopores over time. Hence, this method provides a means to induce and investigate the formation of nanoprecipitates or nanoparticles. Interestingly, precipitates with a slight electric charge were cleared from the pore, causing the conductance of the pore to return to its original value. This process repeated, resulting in stable oscillations of the ionic current. Although such oscillations might be useful in fluidic logic circuits, few conditions capable of generating oscillations in ionic currents have been reported. The frequency and amplitude of oscillations could be tuned by changing the concentration of the precipitating molecule, the $\mathrm{pH}$ of the electrolyte, and the applied potential bias. In addition to generating oscillations, nanopores that separate two different solutions may be useful for monitoring and mediating chemical reactions in the mixing zone between two solutions.
\end{abstract}

(Some figures in this article are in colour only in the electronic version)

\section{Introduction}

Nanopores fabricated in synthetic materials are attracting increasing interest for the detection of individual molecules or molecular complexes [1-19], separation of molecules from each other [20], generation of electrical potentials in a bioinspired approach [21], and studying selective transport in confined environments $[17,19]$. Nanopores in nature, such as ion channel proteins, are involved in essential physiological processes ranging from the regulation of membrane potentials and cell volume to sustaining an immune response [22, 23]. Moreover, a few charged atoms in the interior of biological and synthetic nanopores can have dramatic effects on the permeation of ions $[24,25]$. These effects have been applied to generate single-molecule sensors as well as fluidic diodes [10, 11, 13, 16, 19, 26-29]. Moreover, the nanoscale dimensions of these pores permit studying molecular transport in femtoliter volumes as well as interactions between synthetic materials and hydrophobic, polar or charged molecules at the nanoscale [25, 30-34]. Typically these investigations proceed by measuring ionic currents through a nanopore as a function of an applied potential bias. The frequency, duration, and amplitude of transient variations in the ionic current, caused by the translocation of analytes through the pore, can provide single-molecule information about the concentration, charge and molecular volume of analytes. 
Recently, Siwy's group reported the occurrence of oscillatory currents induced by the precipitation of salts $\left(\mathrm{CaHPO}_{4}, \mathrm{CoHPO}_{4}, \mathrm{Co}(\mathrm{OH})_{2}\right.$ and $\left.\mathrm{Mg}(\mathrm{OH})_{2}\right)$ within the pore [35-37]. The authors demonstrated that an applied potential bias greater than $0.2 \mathrm{~V}$ coupled with the accumulation of ions inside the pore (due to charges on the nanopore surface) resulted in the dynamic precipitation of these salts with concomitant oscillations in ionic current.

Oscillations in the ionic current through the pore indicate that the electrochemical processes within the pore are far from equilibrium, and thus, are an example of a nonlinear chemical system [35]. Such nonlinear chemical systems are rare, fundamentally interesting, and may have unique applications [35]. For instance, ionic current oscillations induced by the precipitation of molecules at the nanoscale may provide a means to induce and investigate the formation of nanoprecipitates as well as to monitor the kinetics of precipitation. Nanoscale precipitates may be useful for (i) determining solubility constants or solubility products, (ii) monitoring crystallization processes, and (iii) formulating nanoprecipitates [38] for pharmaceutical formulations or other technical applications.

Here, we explored the possibility of generating ionic current oscillations by precipitating organic molecules in a nanopore since nanoscale precipitates from organic drug molecules are relevant for the pharmaceutical industry [39]. We were also interested in monitoring the physicochemical processes in the mixing zone between two solutions. For this purpose we set up a nanopore to connect a predominantly organic solvent with an aqueous solvent. We employed a hydrophobic molecule, Tram-34 (a small molecular weight drug that blocks the potassium ion channel $\mathrm{K}_{\mathrm{Ca}} 3.1$ ) [40], which has a relatively high solubility in organic solvents (here dimethylsulfoxide) and a relatively low solubility in aqueous solutions. Dissolving Tram-34 at millimolar concentrations in the organic solution on one side of the pore led to its precipitation inside the nanopore in the mixing zone between the organic solution and the aqueous solution. Surprisingly, this system not only led to detectable reductions in current through the pore due to precipitation, it also displayed oscillatory behavior. Precipitation was followed by the clearance of the precipitate out of the pore followed by the next cycle of precipitation and clearance. To explore the mechanisms of the current oscillations due to the precipitating organic molecule, we characterized these ionic current oscillations as a function of the size of the pore, the concentration of the precipitating molecule, the charge of the molecule, and the applied potential bias across the pore.

\section{Results}

\subsection{Ionic current oscillations induced by periodic precipitation of Tram-34 in the mixing zone between two solutions inside a conical nanopore}

We identified the ion channel blocker Tram-34 as a molecule that may precipitate in the mixing zone between the two solutions due to its relatively high solubility $(10-50 \mathrm{mM})$ in

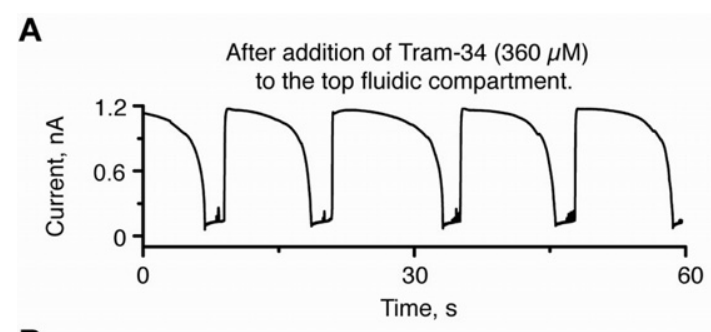

B

Without Tram-34 in the top fluidic compartment.
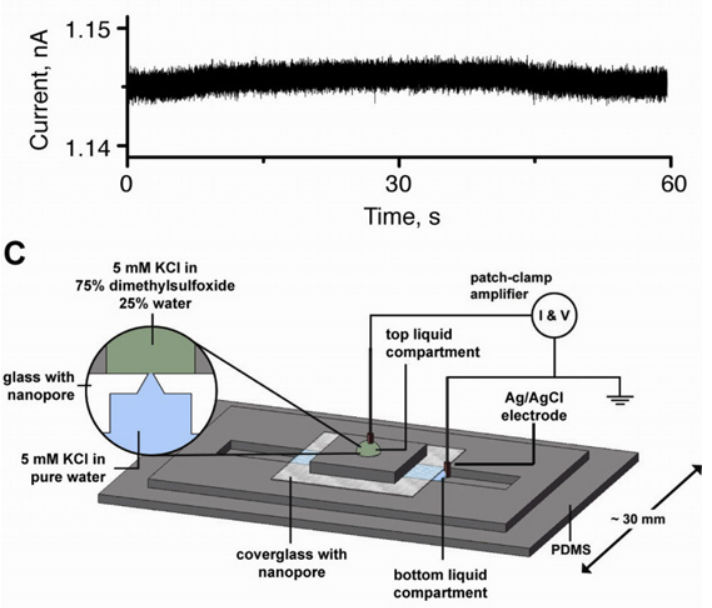

Figure 1. Oscillations of ionic current induced by periodic precipitation of Tram-34 in the mixing zone between two solutions inside a nanopore and a schematic illustration of the recording setup with the two solution compartments. Current traces were recorded at an applied potential bias of $+0.4 \mathrm{~V}$ with (A) or without (B) Tram-34 in top liquid compartment. (C) Schematic diagram of the experimental setup used to record ionic currents through nanopores. Two fluidic compartments were created by mounting a chip that contained a nanopore between two pieces of polydimethylsiloxane (PDMS). The top fluid compartment was filled with a solution containing $5 \mathrm{mM} \mathrm{KCl}$ in $75 \%(\mathrm{v} / \mathrm{v})$ dimethylsulfoxide and $25 \%$ $(\mathrm{v} / \mathrm{v})$ water while the bottom compartment was filled with $5 \mathrm{mM}$ $\mathrm{KCl}$ in pure water. Currents were recorded through a conical nanopore with a tip diameter of $500 \mathrm{~nm}$ that was fabricated in borosilicate cover glass.

dimethylsulfoxide and its low solubility $(\sim 2 \mu \mathrm{M})$ in aqueous solutions. One of the two amines in Tram-34 has a $\mathrm{pKa}$ value of $\sim 0.4$ and therefore at neutral $\mathrm{pH}$, approximately 1 out of every 6.3 million molecules are positively charged (due to the aromatic character of Tram-34, we expect the second amine to have a $\mathrm{p} K$ a value even smaller than 0.4 ). In order to carry out resistive-pulse recordings of the precipitation of Tram-34 inside of pores as shown in figures 1(A) and (B), we prepared the organic solution such that it contained $5 \mathrm{mM} \mathrm{KCl}$ in $75 \%(\mathrm{v} / \mathrm{v})$ dimethylsulfoxide and $25 \%(\mathrm{v} / \mathrm{v})$ water and filled the top fluidic compartment of the recording setup with this solution. The bottom fluidic compartment contained a purely aqueous solution, with $5 \mathrm{mM} \mathrm{KCl}$ (figure 1(C)). To connect these two solutions, we used a conical nanopore fabricated in borosilicate cover glass with a tip diameter of $500 \mathrm{~nm}$ (figure 1) [2, 41, 42] or a cylindrical nanopore fabricated in silicon nitride with a diameter of $92 \mathrm{~nm}$ and a length of $275 \mathrm{~nm}$. To induce precipitation in the mixing zone between the two 


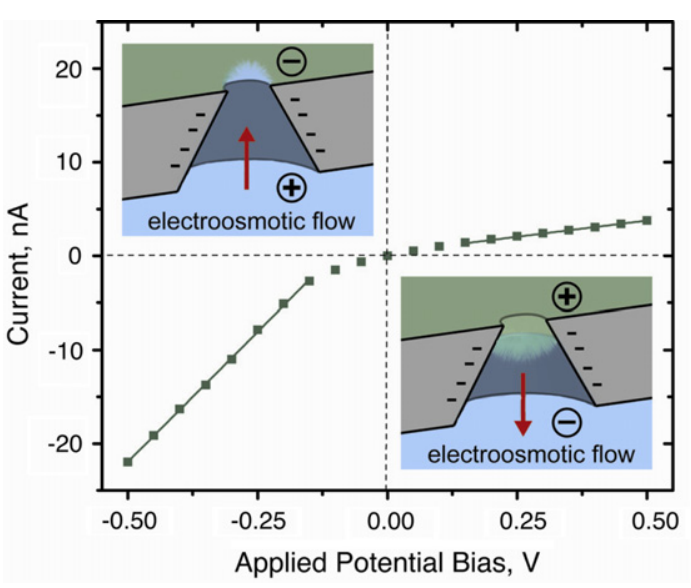

Figure 2. Current versus voltage plot showing ion current rectification as a result of electroosmotic flow and the different conductance of the two solutions described in figure 1(B). The insets illustrate the effects of electroosmotic flow, which depending on the polarity of the potential bias, filled the narrowest constriction of the pore with either the aqueous solution of relatively low viscosity (high conductance) or with the predominantly organic dimethylsulfoxide solution of relatively high viscosity (low conductance). Note that dimethylsulfoxide and water are miscible generating a mixing zone in the pore. Currents were recorded under the same conditions as in figure 1 .

solutions, we added Tram-34 to the organic dimethylsulfoxide solution in the top fluidic compartment and applied a potential bias of $+0.4 \mathrm{~V}$ to the top compartment relative to the bottom compartment (the bottom compartment was always electrically grounded). Figure 1(A) illustrates that this configuration led to remarkably regular, large-amplitude oscillations in the recorded ionic current. In contrast, figure 1(B) shows stable baseline currents recorded in the absence of Tram-34.

Although we expected to observe a reduction of the baseline current due to the precipitation of Tram-34 inside the nanopore, the oscillations shown in figure 1(A) were surprising. Remarkably, tens of seconds after the current reached a value of only $15 \%$ of its initial value, it returned rapidly (within 1-200 $\mathrm{ms}$ ) to the original value indicating that the pore somehow reopened periodically. These current oscillations persisted over the entire course of the experiment (sometimes for several hours).

Interestingly, we only observed these ionic current oscillations at applied potential biases of positive polarity. We attribute this result to the direction of the electroosmotic flow in the pore, which was directed from the top compartment toward the bottom compartment at a positive polarity, see figure 2 [43]. We previously confirmed that this electroosmotic effect can result in the ion current rectification shown in figure 2 due to the different viscosities, and hence, resistivities of the two solutions [43]. In the work presented here, electroosmotic flow filled the narrowest constriction of the pore with the solution from the top compartment when the top compartment was biased positively. This solution contained Tram-34 and induced a detectable decrease in the current due to precipitation of Tram-34 inside the pore.
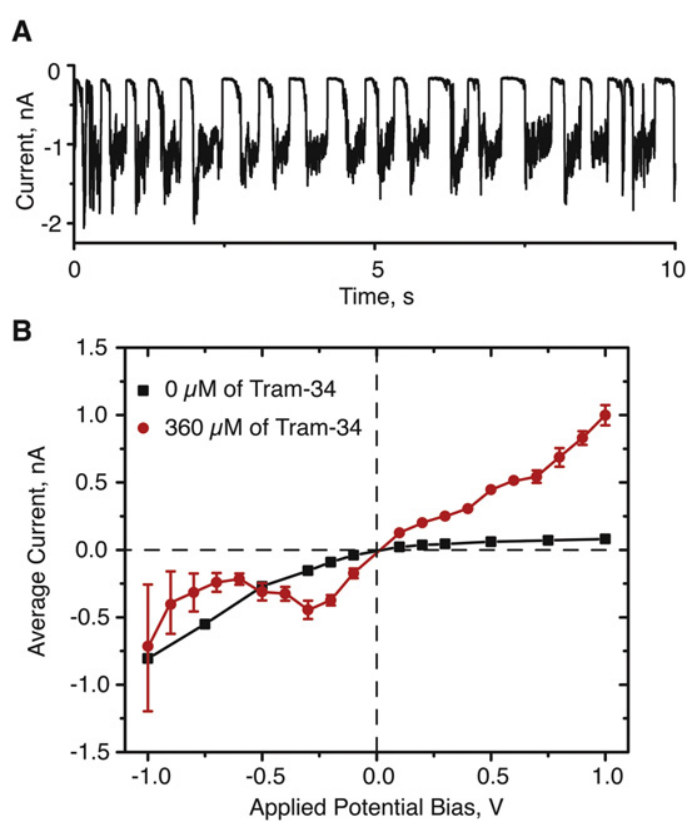

Figure 3. Current oscillations in a nanopore with a diameter of $92 \mathrm{~nm}$ induced by the precipitation of Tram-34 and reversal of the electroosmotic flow after the addition of Tram-34. (A) Ionic current oscillations recorded at $-1.0 \mathrm{~V}$ after the addition of $360 \mu \mathrm{M}$ of Tram-34 to the top solution compartment. (B) Plot of average current versus applied potential bias showing ion current rectification at different polarities depending on the presence or absence of Tram-34 in the top compartment. In the absence of Tram-34, the conductance at negative potential biases was higher than at positive potential biases, while in the presence of Tram-34, the conductance was higher at positive potential biases that at negative potential biases. The solutions were the same as described in figure 1 .

\subsection{Reducing the diameter of the nanopore increased the frequency of ionic current oscillations}

To determine if the dimensions of a pore influence the frequency of the ionic current oscillations, we repeated the experiment described in figure 1 with a cylindrical nanopore in silicon nitride with a diameter of $92 \mathrm{~nm}$ and a length of $275 \mathrm{~nm}$. Due to its small diameter, we expected this nanopore to be blocked by a precipitate at a faster rate than the pore shown in figure 1. Figure 3(A) shows that the same concentration of Tram-34 resulted in ionic current oscillations at an approximately 20 times greater frequency than those observed with the $500 \mathrm{~nm}$ pore in figure 1(A). Interestingly, in the case of the $92 \mathrm{~nm}$ pore in silicon nitride, the ionic current oscillations occurred at a negative applied potential bias of $-1.0 \mathrm{~V}$ and not at an applied potential bias of positive polarity.

\subsection{Adsorption of Tram-34 to pores in silicon nitride led to the reversal of electroosmotic flow}

To understand the occurrence of oscillations at negative applied potential biases with pores in silicon nitride, we plotted average ionic currents through this pore as a function of the applied potential bias before and after the addition of Tram-34 (figure 3(B)). In the absence of Tram-34, we observed a larger resistance under positive potential biases compared to negative 
potential biases. This result indicates that the electroosmotic flow was directed from the more viscous, and hence more resistive, solution in the top compartment toward the bottom compartment [43]. The direction of the electroosmotic flow indicates that the wall of the pore in silicon nitride displayed a net negative charge in the absence of Tram-34. Figure 3(B) shows that after the addition of Tram-34 the larger resistance occurred at potential biases with the opposite polarity (i.e. at negative potential biases). This result indicates a reversal of the electroosmotic flow, which would be expected for a net positive charge on the walls of the pore. Additional evidence for this reversal of the direction of electroosmotic flow stems from the resistance of the pore: under negative potential biases and before the addition of Tram-34 the resistance through the pore $(R=1.05 \pm 0.08 \mathrm{M} \Omega)$ was similar to the resistance of the pore under positive potential biases after the addition of Tram-34 ( $R=0.98 \pm 0.07 \mathrm{M} \Omega)$ [43]. We and Martin's group demonstrated previously that neutral or charged drugs can adsorb to the walls of a nanopore and change the charge on the walls of the pore. This adsorption can in turn affect the magnitude or direction of electroosmotic flow as well as the permeation of ions [43, 44]. Consequently, we attribute the reversed direction of the electroosmotic flow to adsorption of Tram-34 molecules to the pore walls. Due to the presence of a small fraction of Tram-34 molecules with a positive charge, this adsorption could impart a positive charge on the walls of the pore and reverse the direction of the electroosmotic flow. The reversed direction of the electroosmotic flow due to the adsorption of Tram-34 had the consequence that negative potential biases were required to fill the nanopore with the solution from the top compartment, and consequently, facilitate the transport of Tram-34 into the nanopore. Therefore, we observed oscillations only at negative potential biases in contrast to experiments in glass, which showed oscillations only at positive potential biases. In pores fabricated in glass, the presence of Tram-34 did not reverse the direction of the electroosmotic flow. We attribute this behavior to the significantly greater density of negative charges on glass compared to silicon nitride $[45,46]$. The results in figures 1 and 3 suggest that sub-monolayer coverage of the glass surface with Tram-34 led to a surface with a net negative charge while it led to a net positive charge on the silicon nitride surface.

\subsection{Ionic current oscillations increased in frequency with increasing concentrations of Tram-34}

To investigate the effect of the concentration of Tram-34 on the frequency of ionic current oscillations, we titrated increasing amounts of Tram-34 into the predominantly organic solution in the top fluidic well. Figures 4(A) and (B) shows that the frequency of ionic current oscillations increased with increasing concentrations of Tram-34. At concentrations of Tram-34 greater than $0.63 \mathrm{mM}$, current oscillations occurred at two superimposed frequencies (figures $4(\mathrm{~A})$ and (E)). For instance, in the presence of $1.1 \mathrm{mM}$ Tram-34 in the top fluid compartment, oscillations occurred at a frequency of $0.5 \mathrm{~s}^{-1}$ and at a frequency of $0.015 \mathrm{~s}^{-1}$ (bottom current trace in figure $4(\mathrm{~A})$ ).
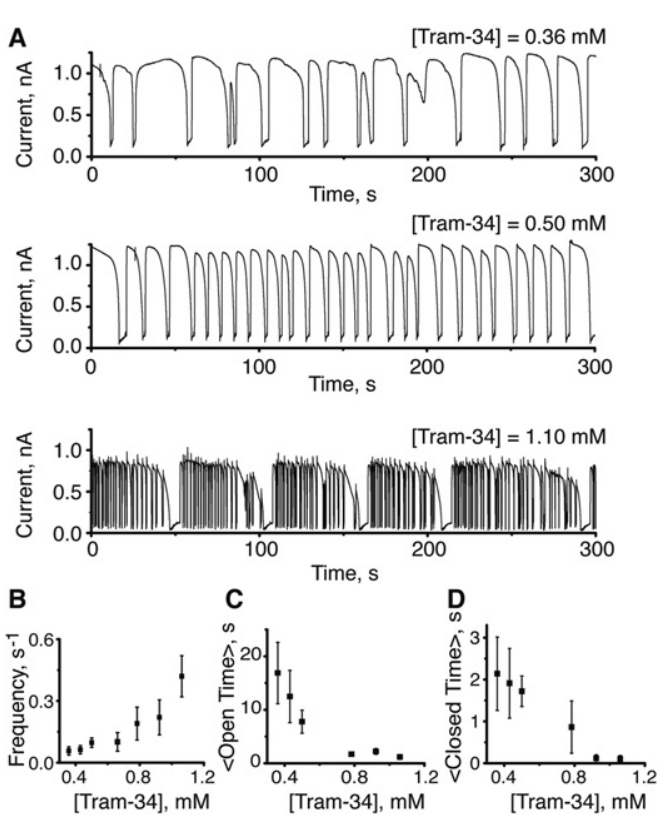

E
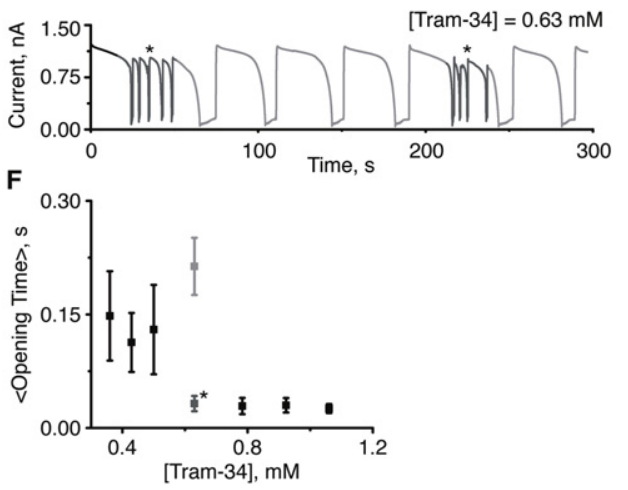

Figure 4. Ionic current oscillations induced by precipitates of Tram-34 in the mixing zone between two solutions inside a nanopore. (A) Current traces recorded at an applied potential bias of $+0.4 \mathrm{~V}$ showing ion current oscillations for three concentrations of Tram-34. (B) Plot of the frequency of ionic current oscillations as a function of the concentration of Tram-34 in the top fluid compartment. (C) Plot of the average time interval during which the current through the pore was greater than $50 \%$ of its maximum value as a function of the concentration of Tram-34. (D) Plot of the average time interval during which the pore remained blocked by a precipitate as a function of the concentration of Tram-34. (E) Current trace recorded with a concentration of $0.63 \mathrm{mM}$ Tram-34 in the top fluid compartment. (F) Average opening time (i.e. the time between the end of the closed state and the beginning of the open state) as a function of the concentration of Tram-34. The two grey data points at [Tram-34] $=0.63 \mathrm{mM}$ refer to the types of opening observed in panel $\mathrm{E}$ in which the light grey point corresponds to the low frequency oscillations and the starred, dark grey point corresponds to the high frequency oscillations. The solutions and pore were the same as described in figure 1.

To characterize these ionic current oscillations, we determined the average time that the pore was open, the average time that the pore was closed, and the probability that the pore was closed as a function of the concentration of Tram34. Figures $4(\mathrm{C})$ and (D) show that the average time the pore was open (defined by current values greater than $50 \%$ of the maximum current) and was closed (defined by current values less than $15 \%$ of the maximum current) decreased with increas- 
A

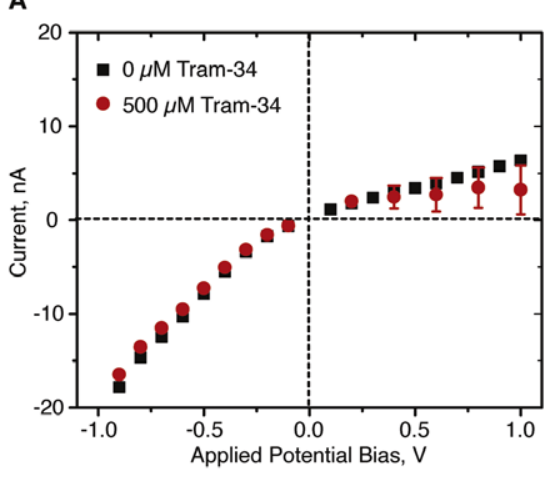

C

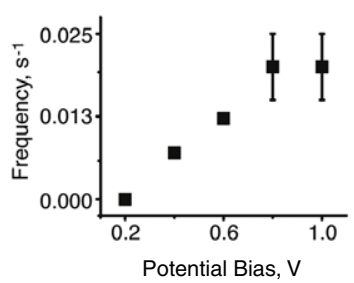

D

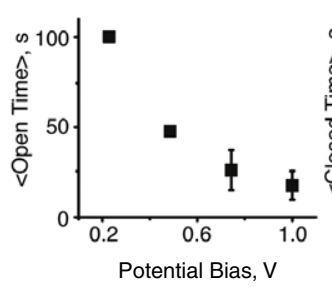

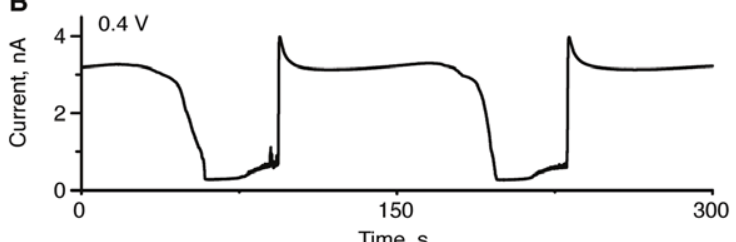

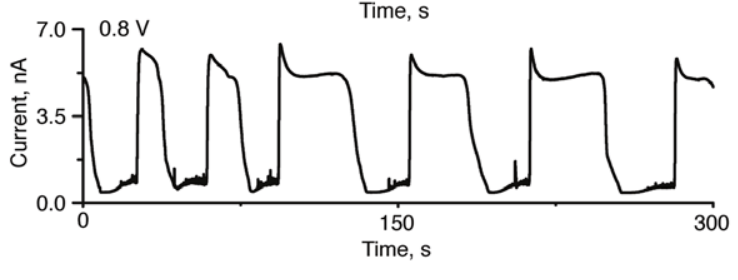

E

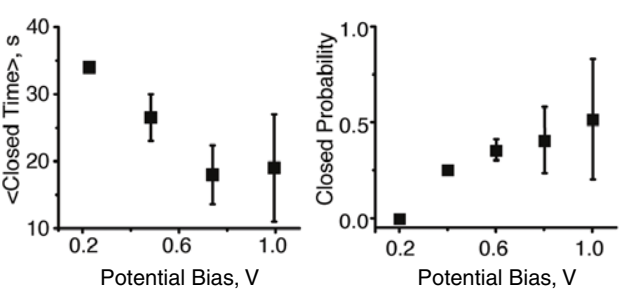

Figure 5. Characteristics of ionic current oscillations as a function of the applied potential bias. (A) Currents as a function of the applied potential bias in the absence and in the presence of $0.5 \mathrm{mM}$ Tram-34 in the top compartment. (B) Original current traces recorded in the presence of $0.5 \mathrm{mM}$ Tram-34 at an applied potential bias of +0.4 and $+0.8 \mathrm{~V}$. Plots of the frequency of ionic current oscillations (C), the average time interval during which the current through the pore was greater than $50 \%$ of its maximum value (D), the average time interval during which the pore remained blocked by a precipitate (E), and the closed probability (F), as a function of the applied potential bias. The solutions were the same as described in figure 1; the conical pore was fabricated in glass with a tip diameter of 640 nm.

ing concentrations of Tram-34. From this data, we calculated the probability that the pore was closed and determined that it was independent of the Tram-34 concentration with an average value of $9.4 \pm 5 \%(N=6)$. In other words, the pore was open $\sim 90 \%$ of the time and closed $\sim 10 \%$ of the time, independent of the concentration of Tram-34.

To determine whether the time required for the pore to reopen was dependent on the concentration of Tram-34, we measured the time between the end of the closed state and the beginning of the open state for at least 20 current oscillations. Surprisingly, the opening of the pore occurred over two timescales differing by an order of magnitude; figure 4(F) shows that at Tram-34 concentrations less than $0.63 \mathrm{mM}$, the time required to reopen the pore was independent of the concentration of Tram-34 and occurred over $\sim 0.15 \mathrm{~s}$ while at concentrations greater than $0.63 \mathrm{mM}$, the reopening occurred over $\sim 0.03 \mathrm{~s}$. Interestingly, figure 4 (E) shows current oscillations recorded in the presence of $0.63 \mathrm{mM}$ Tram-34 with reopening of the pore occurring over both timescales. The low frequency oscillations shown in figure 4(E) in light grey have opening times of $0.2 \mathrm{~s}$ while the high frequency oscillations shown in dark grey and marked with a star have opening times of $\sim 0.03 \mathrm{~s}$.

\subsection{The frequency of ion current oscillations increased with increasing applied potential bias}

We hypothesized that with an increased applied potential bias, and hence increased electroosmotic flow of the solution from the top fluidic compartment into the pore, the frequency of ionic current oscillations would increase as a consequence of increased transport of Tram-34 into the pore [47-49]. Figure 5(A) shows the average ionic current through the pore for various potential biases, and figures $5(\mathrm{~B})$ and (C) show that, as expected, the frequency of ionic current oscillations increased with increasing applied potential bias, when a threshold of $+0.4 \mathrm{~V}$ was exceeded. Furthermore, figure 5(D) shows that the average time interval during which the pore remained open decreased with increasing applied potential biases; similarly, figure 5(E) shows that the average time interval during which a precipitate blocked the pore also decreased with increasing applied potential biases. In contrast to the dependence of current oscillations on the concentration of Tram-34, figure 5(F) shows that the probability for a closed pore increased with increasing applied potential bias, and the time required for the pore to transition from the closed state to the open state $(0.5 \pm 0.2 \mathrm{~s})$ was independent of the applied potential bias.

\subsection{Charged molecules are required to generate oscillations of ionic current}

To investigate the effect of the partial charge of Tram-34, we compared the ionic current oscillations induced by Tram-34 in HEPES buffered solutions with $\mathrm{pH}$ values of $6.2,7.2$ or 8.2. One of the two amines in Tram-34 has a $\mathrm{p} K$ a value of $\sim 0.4$, and hence, in the solution with a $\mathrm{pH}$ of 6.2 approximately 1 of every 0.63 million Tram-34 molecules were positively charged. While in the solution with a $\mathrm{pH}$ of 7.2 approximately 1 of every 6.3 million Tram-34 molecules were positively charged, and in the solution with a $\mathrm{pH}$ of 8.2 approximately 1 of every 63 million Tram-34 molecules were positively charged. 


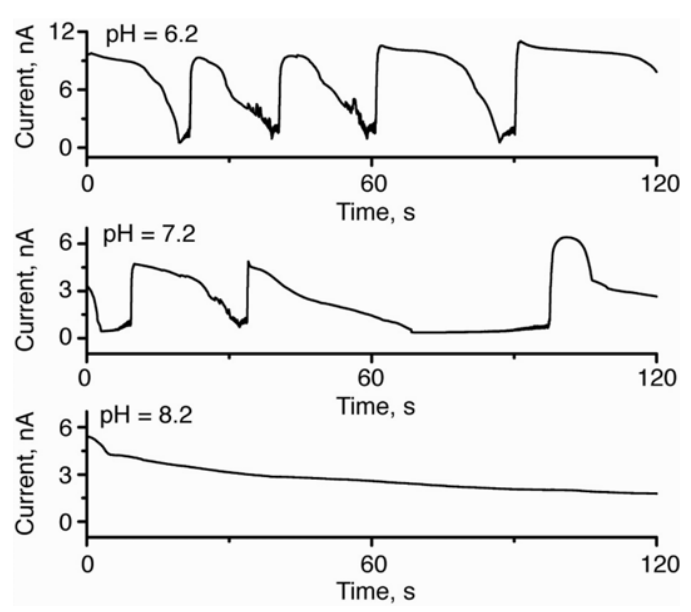

Figure 6. Influence of the $\mathrm{pH}$ on the frequency of oscillations in the current due to periodic precipitation of Tram-34 in the pore. Current traces were recorded with a conical pore with a tip diameter of $500 \mathrm{~nm}$ fabricated in borosilicate cover glass and at an applied potential bias of $+0.7 \mathrm{~V}$. The solutions contained $5 \mathrm{mM}$ of HEPES buffer.

Figure 6 shows that current oscillations occurred at the highest frequency in the solutions with a $\mathrm{pH}$ of 6.2 compared to the solutions with a $\mathrm{pH}$ of 7.2 or 8.2 . At a $\mathrm{pH}$ value of 8.2 , we observed a slow (over 2-3 min) decrease in the ionic current due to the precipitation of Tram-34; in this case, we did not observe the reopening of the pore. To confirm that the $\mathrm{pH}-$ dependence of the ionic current oscillations was due to the change in the fraction of charged Tram-34 molecules and not to an artifact of the HEPES buffer, we repeated these experiments with solutions prepared with phosphate buffer; again, ionic current oscillations occurred with the highest frequency in the solutions with a $\mathrm{pH}$ of 6.2 and none occurred in the solutions with a $\mathrm{pH}$ of 8.2. This result suggests that the precipitate of Tram-34 must contain at least a few positive charges in order for the nanopore to reopen. Assuming spherical molecules of Tram-34 with a volume of $308 \AA^{3}$ [40] pack with hexagonal closest packing into a spherical precipitate at the tip of the pore with a diameter of $500 \mathrm{~nm}$, approximately 1.28 million Tram-34 molecules would be expected on the surface of the precipitate particle. Thus, in solutions with a $\mathrm{pH}$ of 6 , this precipitate would be expected to have an average of $\sim 2$ positive charges on its surface, while in solutions with a $\mathrm{pH}$ of 8 the precipitate would have an average of only $\sim 0.02$ positive charges on its surface. These considerations are consistent with the results in figure 6 and indicate, but do not prove, that the $\mathrm{pH}$ of the solutions could influence the ejection of precipitates of Tram-34 from a pore with an applied electric field along its length axis.

Changes in the $\mathrm{pH}$ of the electrolyte also affect the density of negative charges on the surface of the glass pore, and as a result, we cannot exclude the possibility that changes in the density of negative charges on the surface of the pore during this experiment may have affected the signature of the ionic current oscillations. We expect, however, that as the $\mathrm{pH}$ of the electrolyte decreased from 8.2 to 6.2 , the increased frequency of ionic current oscillations and the clearance of

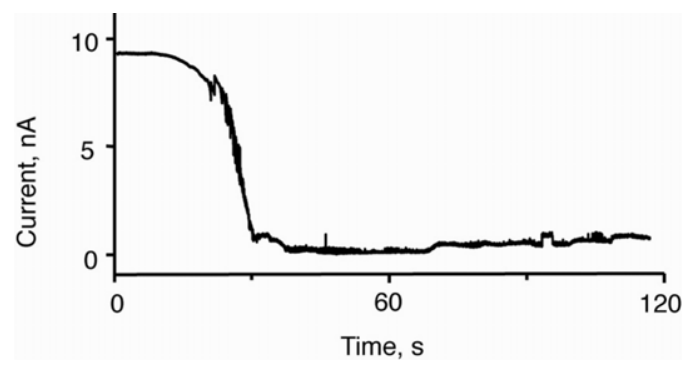

Figure 7. Reduction in ionic current due to the precipitation of felodipine, an electrically neutral molecule, in a pore. The current trace was recorded at an applied potential bias of $+0.7 \mathrm{~V}$ after the addition of $0.63 \mathrm{mM}$ of felodipine to the top compartment. Both solutions contained $5 \mathrm{mM} \mathrm{KCl}$ and $5 \mathrm{mM}$ HEPES at $\mathrm{pH}$ 7.2.

the precipitate from the pore (in electrolytes with $\mathrm{pH} \leqslant 7.2$ ) was primarily due to the 100 -fold increase in the fraction of positively charged Tram-34 molecules since the density of negative charges on the glass surface decreases only by $\sim 5$-fold over this range of $\mathrm{pH}$ values $[46,50]$.

To provide additional evidence that the charge of the precipitating molecule was primarily responsible for the clearance of the precipitate from the pore, we attempted to observe ionic current oscillations with the electrically neutral molecule felodipine. The solubility of felodipine in aqueous solutions is $\sim 50 \mu \mathrm{M}$, and therefore, we expected this molecule to precipitate in the mixing zone between the two solutions in the pore when the dimethylsulfoxide solution on top of the chip contained a relatively high concentration of felodipine. Figure 7 shows that in the presence of $630 \mu \mathrm{M}$ felodipine in the top compartment the current through the pore decreased by more than $85 \%$, and the pore remained blocked by the felodipine precipitate during the entire course of the experiment. This result suggests, not surprisingly, that the mechanism of precipitation can also occur with uncharged molecules. It also suggests that precipitates of organic molecules with low aqueous solubility must contain a minimum charge to be removed from the pore and to generate oscillations in the ionic current.

To examine the effect of the electrostatic interaction between charged molecules and the charge of the pore, we performed three experiments with a negatively charged molecule, indomethacin ( $\mathrm{p} K \mathrm{a}=4.5$ and a solubility in water of $11 \mu \mathrm{M}$ at $\mathrm{pH}$ 1.2) [51], and with pores of different surface charge (i.e. negative, neutral, or positive). In solutions with a $\mathrm{pH}$ of 3 and with an unmodified, negatively charged pore in glass (conical tip diameter of $300 \mathrm{~nm}$ ), the addition of indomethacin to the top compartment caused a slow decrease in the magnitude of the total current to values that were $\sim 75 \%$ of the original current over $\sim 1 \mathrm{~h}$. We obtained nearly identical results using the same pore at $\mathrm{pH}=1$, which neutralized the majority of the negatively charged silanol groups of the glass pore $[52,53]$. The results at $\mathrm{pH}$ values below the $\mathrm{pKa}$ of indomethacin suggest the predominantly neutral molecules of indomethacin slowly adsorbed to the walls of the unmodified pore. We next modified the same pore with polyethylenimine such that the pore exposed positive charges on its surface. Using this modified pore and solutions with a 
$\mathrm{pH}$ of 3 , we observed transient oscillations in the ionic current when concentrations of indomethacin exceeded $1.4 \mathrm{mM}$ in the top compartment and when the applied potential bias was between -0.5 and $-1.0 \mathrm{~V}$. Several periods of oscillations occurred that persisted for 30-60 s with a frequency of $0.05-$ $0.43 \mathrm{~s}^{-1}$. This result suggests that electrostatic attractions between the positively charged pore walls and the small fraction of negatively charged indomethacin molecules, as well as electrophoretic forces acting on the charged precipitate, were involved in the occurrence of oscillations.

\section{Discussion}

This paper introduces the possibility to monitor the precipitation of organic molecules with low aqueous solubility in a nanopore. These precipitates almost completely blocked the permeation of ions through the pore and under certain conditions these precipitates were cleared from the pore leading to oscillations in the current. A significant question that remains is: what process governs the reopening of the pore? Possible mechanisms include the periodic ejection of the precipitate from the pore or the periodic dissolution of the precipitate as observed previously with inorganic salts by Siwy and co-workers.

Siwy's group reported ionic current oscillations in pores due to precipitation of inorganic salts. In that system, the authors theorized that the precipitation of salts and the subsequent dissolution of these precipitates were responsible for the ionic current oscillations [35]. The precipitation of the salts was found to be a consequence of the charged surface of the pore and the applied potential bias, which resulted in an accumulation of these salts in the pore to concentrations two to three orders of magnitude greater than their solubility product [35]. Consistent with this mechanism of precipitation, the authors proposed that the dissolution of the precipitate occurred because, in the presence of a precipitate, the applied potential bias dropped over the length of the precipitate rather than over the entire length of the pore. Consequently, the concentrations of ions in the solution near the pore decreased to values below the solubility product permitting the dissolution of the precipitate and the reopening of the pore [35].

The processes governing the precipitation of the organic molecules in the work presented here and the subsequent reopening of the pore may be different than in the system reported by Siwy's group, since here the precipitation of organic molecules in the pore is governed by the different solubility of Tram-34 in the two different solutions on either side of the pore. We propose that the precipitation begins on the walls of the pore and in the mixing zone between the two solutions because these organic molecules have a very low solubility in the aqueous solution. Evidence for the nucleation of the precipitate on the pore walls includes the slow reductions in current over tens of seconds and the reversal of the direction of electroosmotic flow in silicon nitride pores after addition of organic molecules that contain a very small fraction of molecules with a charge opposite to that of the pore wall.
Based on a mechanism of precipitation different from the work presented by Siwy and colleagues, a possible explanation for reopening of the pore in the work presented here could be the ejection of the precipitate from the pore due to the applied electric field rather than due to the dissolution of the precipitate. We observed that the average duration during which the pore was closed decreased with increasing concentrations of Tram-34 (figure 4(D)). This result would not be expected if the reopening of the pore were governed by the dissolution of the precipitate. Furthermore, the average duration during which the pore was closed also decreased with increasing electric fields (figure $5(\mathrm{E})$ ). This observation would be consistent with the ejection of the precipitate from the pore due to electrophoretic or electroosmotic forces acting on the precipitate. Finally, we observed that electrically neutral molecules such as felodipine precipitated in the pore but were never removed from the pore (figure 7). Similarly, in solutions with high $\mathrm{pH}$ values that further reduced the fraction of Tram34 molecules with positive charge, a precipitate formed in the pore but was never cleared from the pore. In contrast, in solutions with low $\mathrm{pH}$ values, Tram-34 precipitates in the pore had a slight positive charge, and these precipitates were cleared from the pore in an oscillatory fashion. Together these results suggest that an electrophoretic force on the precipitate due to the applied electric field and its small partial charge may be required to force the precipitate from the pore.

In contrast to this theory and similar to recent results obtained by Siwy's group [37], we observed that the time for the pore to transition from the closed state to the open state did not depend on the magnitude of the applied potential bias. If the reopening of the pore would depend on an electrophoretic force on the particle, we would expect the time for the precipitate to move out of the pore to decrease with increasing electrophoretic force due to the electric field strength. Hence, this particular result suggests that electroosmotic or electrophoretic forces may not have been responsible for the reopening of the pore and favors the theory that precipitates dissolved rapidly after their formation.

We cannot rule out the possibility that both dissolution and electrophoretic forces play a role in the clearance of the pore. The observation that electrically neutral molecules precipitated by apparently the same mechanisms as slightly charged molecules while never clearing the pore in the same manner as charged precipitates, suggests that an electrophoretic force on the precipitate plays some role in reopening the pore, possibly by ejecting the precipitate electrophoretically from the pore.

\section{Conclusion}

This paper demonstrates a method to generate ionic current oscillations with various frequencies and amplitudes based on the concentration of Tram-34 and the applied electric field. These oscillations could be useful in areas of fluid electronics and fluid logic circuits, in which oscillations in ionic current remain unusual, in contrast to the phenomena of ion current rectification $[19,35,54]$. 
Furthermore, we demonstrated ionic current oscillations induced by the precipitation of organic molecules, including therapeutic drugs, with low aqueous solubility. The solubility of drugs is one of the first properties determined for new drug candidates because it is related to their bioavailability; the low aqueous solubility of many drug candidates is a key reason for excluding them from further trials [39]. Hence, rapid methods to determine the solubility of small molecules are useful for the pharmaceutical industry [39]. We propose that with adequate models to describe the mixing of the two solutions within the nanopore, this technique could yield information about the solubility of drug molecules. In addition, many of the molecules being screened to determine their solubility are early in the discovery process, and may therefore, be available in limited quantities and may be expensive [55]. Methods of determining solubility with minimal amounts of the drug candidate and reagent volumes would be beneficial for the field of drug discovery. The technique we presented here could be integrated with microfluidics to reduce reagent volumes below microliters.

We demonstrated that precipitates with a slight net charge could, under certain conditions, be removed from the nanopore in an oscillatory fashion. This unusual characteristic permitted the precipitation process to repeat indefinitely. Further investigations may make it possible to understand the processes governing the reopening of the pore completely. If the reopening of the pore entails the ejection of a formed precipitate, future techniques may make it possible to produce and collect nanoprecipitates formed from drug molecules. These nanoprecipitates may replicate the size and shape of the nanopore, and hence, have a well defined, predetermined shape. Suspensions of nanoprecipitates may be of interest to the pharmaceutical industry since the size of the precipitate affects the rate of dissolution and release of the drug from the precipitate. Currently, the smallest nanoprecipitates formed from drug molecules are about 100-200 nm in diameter [56]. With fabricated nanopores that have diameters smaller than $100 \mathrm{~nm}$, this technique could potentially produce nanoprecipitates of drug molecules with diameters smaller than $100 \mathrm{~nm}$.

Finally, the work described here employed a nanopore that connected two solutions of different solvent character, and thus, generated a mixing zone between these solutions inside the pore. Since processes that occur inside a nanopore can typically be detected by a change in the ionic current, chemistry that occurs at the interface between two solutions could potentially be explored and monitored at the nanoscale and in real time. For example, the formation of nylon occurs at the interface between an aqueous solution and a solution of hexane. To exploit nanopores for examining processes occurring between two solutions, accurate, electrochemical models with high resolution in time will be required to describe the mixture of electrolytes and reagents in the pore [35].

\section{Materials and methods}

\subsection{Solutions and materials}

To obtain conducting solutions with different solvent characteristics, we prepared a solution of $5 \mathrm{mM}$ of potassium chloride $(\mathrm{KCl})$ in pure water, and a solution of $5 \mathrm{mM}$ $\mathrm{KCl}$ in a mixture of $75 \%(\mathrm{v} / \mathrm{v})$ dimethyl sulfoxide (DMSO) and $25 \%(\mathrm{v} / \mathrm{v})$ deionized water $(\rho=18.2 \mathrm{M} \Omega \mathrm{cm}$, Millipore). Where indicated, we also added $5 \mathrm{mM}$ of 4-(2-hydroxyethyl)-1-piperazineethanesulfonic acid (HEPES) buffer or $5 \mathrm{mM}$ of dipotassium phosphate buffer $\left(\mathrm{K}_{2} \mathrm{HPO}_{4}\right)$ to these solutions. We adjusted the $\mathrm{pH}$ value of the solutions to $6.2,7.2$, or 8.2 by adding hydrochloric acid $(\mathrm{HCl})$ or potassium hydroxide $(\mathrm{KOH})$. We purchased Tram-34 (1-[(2-Chlorophenyl)diphenylmethyl]-1H-pyrazole), felodipine (Plendil, 4-(2,3-Dichlorophenyl)-1,4-dihydro-2,6dimethyl-3,5-pyridinecarboxylic acid ethyl methyl ester), and indomethacin (1-(4-Chlorobenzoyl)-5-methoxy-2-methyl3-indoleacetic acid) from Sigma-Aldrich, Inc. (Saint Louis, MO); all molecules were dissolved in pure dimethylsulfoxide for storage. We purchased linear polyethylenimine, M.W. $\sim 25$ kDA from Polysciences Inc. (Warrington, PA).

\subsection{Nano- and micropores}

Following a previously described process, we used a femtosecond pulsed laser followed by an etch with hydrofluoric acid (HF) to fabricate conical pores with tip diameters of 500, 640 and $1000 \mathrm{~nm}$ in borosilicate glass (thickness $\cong 150 \mu \mathrm{m}$, Corning 0211, Fisher Scientific, Pittsburgh, PA) [2, 4, 41, 42]. Prior to each experiment, we cleaned the glass substrates with the conical pores in a freshly prepared, hot mixture of $3: 1(\mathrm{v} / \mathrm{v})$ concentrated sulfuric acid, and $30 \%(\mathrm{v} / \mathrm{v})$ hydrogen peroxide for at least $15 \mathrm{~min}$. Professor Jiali Li, University of Arkansas, AR, kindly provided a second cylindrical pore with a diameter of $100 \mathrm{~nm}$ that was fabricated in a $275 \mathrm{~nm}$ thick silicon nitride membrane [29, 57]. We cleaned these pores in the same fashion as the pores in glass. In order to provide support for the substrates that contained the pores and in order to integrate fluidics, we fabricated a simple poly(dimethylsiloxane) (PDMS, Sylgard 184 Silicone, Dow Corning, Midland, MI) support as shown in figure 1. Where indicated, we generated a pore exposing positive surface charges by incubating the pore in an aqueous solution containing $0.1 \%(\mathrm{w} / \mathrm{w})$ polyethylenimine for $10 \mathrm{~min}$ followed by rinsing the glass chip with deionized water and incubating the dry chip in an oven at $50^{\circ} \mathrm{C}$ for $1 \mathrm{~h}$.

\subsection{Electrical recordings}

We used $\mathrm{Ag} / \mathrm{AgCl}$ pellet electrodes (Warner Instruments, Hamden, CT) to record ion currents. For each recording, we placed the experimental setup in a Faraday cage (Warner Instruments, Hamden, CT) and recorded the electrical current using a patch-clamp amplifier (Axopatch 200B, Molecular Devices Inc.) in voltage clamp mode with the analog lowpass filter set to a cutoff frequency of $2 \mathrm{kHz}$. We used a low noise digitizer (Digidata 1322) with a sampling frequency set to $10 \mathrm{kHz}$ in combination with LabVIEW recording software for all recordings. We performed all data processing using Clampfit 9.2 (Axon Instruments, Union City, CA). At each applied potential difference, we averaged the current over a period of $60 \mathrm{~s}$. All polarities of the applied potential differences refer to the top fluid compartment relative to the bottom fluid compartment, which was always connected to ground. 


\section{Acknowledgments}

This work was supported by a National Science Foundation CAREER Award (MM, Grant No. 0449088). The authors thank Ricardo Capone and Sheereen Majd for valuable discussions. The authors also thank Daniel J Estes and Jeffrey D Uram for their work on the LabVIEW recording software.

\section{References}

[1] Majd S, Yusko E C, Billeh Y N, Macrae M X, Yang J and Mayer M 2010 Curr. Opin. Biotechnol. at press doi:10.1016/j.copbio.2010.05.002

[2] Uram J D, Ke K, Hunt A J and Mayer M 2006 Small 2 967-72

[3] Singer A, Wanunu M, Morrison W, Kuhn H, Frank-Kamenetskii M and Meller A Nano Lett. 10 738-42

[4] Uram J D, Ke K, Hunt A J and Mayer M 2006 Angew. Chem. Int. Edn $\mathbf{4 5} 2281-5$

[5] Uram J D and Mayer M 2007 Biosens. Bioelectron. 22 1556-60

[6] Fologea D, Ledden B, David S M and Li J 2007 Appl. Phys. Lett. 91053901

[7] Talaga D S and Li J L 2009 J. Am. Chem. Soc. 131 9287-97

[8] Sexton L T, Horne L P, Sherrill S A, Bishop G W, Baker L A and Martin C R 2007 J. Am. Chem. Soc. 129 13144-52

[9] Oukhaled G, Mathe J, Biance A L, Bacri L, Betton J M, Lairez D, Pelta J and Auvray L 2007 Phys. Rev. Lett. 98158101

[10] Han A, Creus M, Schurmann G, Linder V, Ward T R, De Rooij N F and Staufer U 2008 Anal. Chem. 80 4651-8

[11] Capone R, Blake S, Restrepo M R, Yang J and Mayer M 2007 J. Am. Chem. Soc. 129 9737-45

[12] Mayer M, Semetey V, Gitlin I, Yang J and Whitesides G M 2008 J. Am. Chem. Soc. 130 1453-65

[13] Blake S, Capone R, Mayer M and Yang J 2008 Bioconjug. Chem. 19 1614-24

[14] Majd S, Yusko E C, Macbriar A D, Yang J and Mayer M 2009 J. Am. Chem. Soc. 131 16119-26

[15] Siwy Z, Trofin L, Kohli P, Baker L A, Trautmann C and Martin C R 2005 J. Am. Chem. Soc. 127 5000-1

[16] Kasianowicz J J, Henrickson S E, Weetall H H and Robertson B 2001 Anal. Chem. 73 2268-72

[17] Gurnev P A, Oppenheim A B, Winterhalter M and Bezrukov S M 2006 J. Mol. Biol. 359 1447-55

[18] Butler T Z, Pavlenok M, Derrington I M, Niederweis M and Gundlach J H 2008 Proc. Natl Acad. Sci. USA 105 20647-52

[19] Howorka S and Siwy Z 2009 Chem. Soc. Rev. 38 2360-84

[20] Siwy Z and Fulinski A 2002 Phys. Rev. Lett. 89198103

[21] Holden M A, Needham D and Bayley H 2007 J. Am. Chem. Soc. 129 8650-5

[22] Hille B 2001 Ion Channels of Excitable Membranes (Sunderland: Sinauer Associaties) pp 347-9

[23] Estes D J, Memarsadeghi S, Lundy S K, Marti F, Mikol D D, Fox D A and Mayer M 2008 Anal. Chem. 80 3728-35
[24] Eisenberg R S 2002 J. Comput. Electron. $1331-5$

[25] Albrecht T, Kornyshev A and Bjornholm T 2008 J. Phys.: Condens. Matter 20370301

[26] Vlassiouk I and Siwy Z S 2007 Nano Lett. 7 552-6

[27] Ali M, Mafe S, Ramirez P, Neumann R and Ensinger W 2009 Langmuir 25 11993-7

[28] Ding S, Gao C L and Gu L Q 2009 Anal. Chem. 81 6649-55

[29] Cai Q, Ledden B, Krueger E, Golovchenko J A and Li J L 2006 J. Appl. Phys. 100024914

[30] Davenport M, Rodriguez A, Shea K J and Siwy Z S 2009 Nano Lett. 9 2125-8

[31] Purnell R F and Schmidt J J 2009 ACS Nano $32533-8$

[32] Schwarz G, Danelon C and Winterhalter M 2003 Biophys. J. 84 2990-8

[33] Van Dorp S, Keyser U F, Dekker N H, Dekker C and Lemay S G 2009 Nat. Phys. 5 347-51

[34] Luan B Q and Aksimentiev A 2010 Soft Matter 6 243-6

[35] Powell M R, Sullivan M, Vlassiouk I, Constantin D, Sudre O, Martens C C, Eisenberg R S and Siwy Z S 2008 Nat. Nanotechnol. 3 51-7

[36] Siwy Z S, Powell M R, Petrov A, Kalman E, Trautmann C and Eisenberg R S 2006 Nano Lett. 6 1729-34

[37] Innes L, Powell M R, Vlassiouk I, Martens C and Siwy Z S 2010 J. Phys. Chem. C 114 8126-34

[38] Guo P, Martin C R, Zhao Y, Ge J and Zare R N 2010 Nano Lett. 10 2202-6

[39] Dressman J and Reppas C 2007 Adv. Drug Deliv. Rev. 59 531-2

[40] Wulff H, Miller M J, Hansel W, Grissmer S, Cahalan M D and Chandy K G 2000 Proc. Natl Acad. Sci. USA 97 8151-6

[41] An R, Uram J D, Yusko E C, Ke K, Mayer M and Hunt A J 2008 Opt. Lett. 53 1153-5

[42] Uram J D, Ke K and Mayer M 2008 ACS Nano 2 857-72

[43] Yusko E C, An R and Mayer M 2010 ACS Nano 4 477-87

[44] Wang J and Martin C R 2008 Nanomedicine 3 13-20

[45] Sonnefeld J 1996 Colloids Surf. A 108 27-31

[46] Behrens S H and Grier D G 2001 J. Chem. Phys. 115 6716-21

[47] Gu L-Q, Cheley S and Bayley H 2003 Proc. Natl Acad. Sci. USA 100 15498-503

[48] Gu L-Q, Cheley S and Bayley H 2003 Biophys. J. 86 293A

[49] Gu L-Q, Cheley S and Bayley H 2004 Biophys. J. 84 483A

[50] Sonnefeld J, Gobel A and Vogelsberger W 1995 Colloid Polym. Sci. 273 926-31

[51] Valizadeh H, Nokhodchi A, Qarakhani N, Zakeri-Milani P, Azarmi S, Hassanzadeh D and Lobenberg R 2004 Drug Dev. Ind. Pharm. 30 303-17

[52] Schmidt C, Mayer M and Vogel H 2000 Angew. Chem.-Int. Edn. 39 3137-40

[53] Siwy Z S 2006 Adv. Funct. Mater. 16 735-46

[54] Siwy Z, Heins E, Harrell C C, Kohli P and Martin C R 2004 J. Am. Chem. Soc. 126 10850-1

[55] Alsenz J and Kansy M 2007 Adv. Drug Deliv. Rev. 59 546-67

[56] Kesisoglou F, Panmai S and Wu Y 2007 Adv. Drug Deliv. Rev. 59 631-44

[57] Li J, Stein D, McMullan C, Branton D, Aziz M J and Golovchenko J A 2001 Nature 412 166-9 